International Journal of Pure and Applied Mathematics

Volume 92 No. 1 2014, 117-124

ISSN: 1311-8080 (printed version); ISSN: 1314-3395 (on-line version)

url: http://www.ijpam.eu

doi: http://dx.doi.org/10.12732/ijpam.v92i1.11

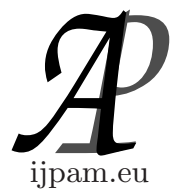

\title{
ON BASES AND MAXIMAL IDEALS IN AN ORDERED SEMIGROUP
}

\author{
Pisan Summaprab ${ }^{1}$, Thawhat Changphas ${ }^{2} \S$ \\ ${ }^{1,2}$ Department of Mathematics \\ Faculty of Science \\ Khon Kaen University \\ Khon Kaen 40002, THAILAND
}

\begin{abstract}
In this paper the concepts of left base, right base and two-sided base of an ordered semigroup are introduced. A sufficient condition for an ordered semigroup contains right bases is given.
\end{abstract}

AMS Subject Classification: $06 \mathrm{~F} 05$

Key Words: semigroup, ordered semigroup, maximal left ideal, right base, two-sided base, covered left ideal

\section{Preliminaries}

Tamura [9] introduced and studied one-sided bases and two-sided bases of semigroups (without order). Fabrici [2] gave a sufficient condition for a semigroup without order contains a right base, and showed that every finite semigroup contains both one-sided bases and two-sided bases. In this paper we introduce the notions of right base, left base and two-sided base of an ordered semigroup, and extend Fabrici's results to ordered semigroups.

Received: January 21, 2014

(C) 2014 Academic Publications, Ltd.

${ }^{\S}$ Correspondence author url: www.acadpubl.eu 
In [1], an ordered semigroup $(S, \cdot, \leq)$ is a semigroup $(S, \cdot)$ together with a partial order $\leq$ that is compatible with the semigroup operation, meaning that, for any $x, y, z$ in $S$,

$$
x \leq y \text { implies } z x \leq z y \text { and } x z \leq y z .
$$

If $A, B$ are nonempty subsets of $S$, we write the set product $A B$ of $A$ and $B$ for the set of all elements $x y$ of $S$ with $x \in A$ and $y \in B$, and write $(A]$ for the set of all elements $x$ of $S$ such that $x \leq a$ for some $a$ in $A$, i.e.,

$$
(A]=\{x \in S \mid x \leq a \text { for some } a \in A\} .
$$

In particular, we write $A x$ for $A\{x\}$. It was shown in [6] that $(A \cup B]=(A] \cup(B]$.

A nonempty subset $A$ of an ordered semigroup $(S, \cdot, \leq)$ is called a left ideal [3] of $S$ if it satisfies the following conditions:

(i) $S A \subseteq A$;

(ii) for any $x \in A$ and $y \in S, y \leq x$ implies $y \in A$.

For $a$ in $S$, the principal left ideal generated by $a$ is $L(a):=(a \cup S a]$. It is known that the union of two left ideals of $S$ is a left ideal of $S$.

A proper left ideal $A$ of an ordered semigroup $(S, \cdot, \leq)$ is called a maximal left ideal if there is no a proper left ideal $L^{\prime}$ of $S$ such that $L \subset L^{\prime}$.

Let $(S, \cdot \leq)$ be an ordered semigroup. The equivalence relation $\mathcal{L}$ is defined by:

$$
a \mathcal{L} b \text { if and only if } L(a)=L(b)
$$

for any $a, b$ in $S$. The $\mathcal{L}$-class containing $a$ in $S$ will be written $L_{a}$. Define a preorder $\preceq$ on the set of all $\mathcal{L}$-classes by:

$$
L_{a} \preceq L_{b} \text { if and only if } L(a) \subseteq L(b)
$$

The symbol $L_{a} \prec L_{b}$ stands for $L_{a} \preceq L_{b}$, but $L_{a} \neq L_{b}$. The symbol $a<b$ stands for $a \leq b$, but $a \neq b$. Note that $a \leq b$ implies $L_{a} \preceq L_{b}$. In particular, $a<b$ implies $L_{a} \prec L_{b}$. 


\section{Ordered Semigroups Containing One-Sided and Two-Sided Bases}

We define one-sided bases and two-sided bases of an ordered semigroup by:

Definition 1. Let $(S, \cdot, \leq)$ be an ordered semigroup. A subset $A$ of $S$ is called a right base of $S$ if it satisfies the following conditions:

(i) $S=(A \cup S A]$;

(ii) if $B$ is a subset of $A$ such that $S=(B \cup S B]$, then $A=B$.

Dually, one can define for $A$ to be a left base of $S$.

By a two-sided base of $S$ we mean a subset $A$ of $S$ such that

(i) $S=(A \cup S A \cup A S \cup S A S]$;

(ii) if $B$ is a subset of $A$ such that $S=(B \cup S B \cup B S \cup S B S]$, then $A=B$.

Example 2. ([7]) Let $(S, \cdot, \leq)$ be an ordered semigroup such that the multiplication and the order relation are defined by:

$$
\begin{aligned}
& \begin{array}{c|lllll}
\cdot & a & b & c & d & e \\
\hline a & a & e & c & d & e
\end{array} \\
& \begin{array}{llllll}
b & a & b & c & d & e
\end{array} \\
& \begin{array}{llllll}
c & a & e & c & d & e
\end{array} \\
& \begin{array}{llllll}
d & a & e & c & d & e
\end{array} \\
& \begin{array}{l|lllll}
e & a & e & c & d & e
\end{array} \\
& \leq=\{(a, a),(b, b),(c, c),(d, d),(e, e),(a, d),(c, e)\} .
\end{aligned}
$$

The covering relation and the figure of $S$ are given by:

$$
\sum_{a}^{d} \quad \int_{c}^{e} \quad \circ b
$$

The left bases of $S$ are $\{a\},\{b\},\{c\},\{d\}$ and $\{e\}$. The right base of $S$ is $\{b, d\}$. $S$ has only one two-sided base: $\{b\}$.

Example 3. ([4]) Let $(S, \cdot, \leq)$ be an ordered semigroup such that the multiplication and the order relation are defined by: 


$$
\begin{aligned}
& \begin{array}{c|ccccc}
\cdot & a & b & c & d & e \\
\hline a & a & a & c & a & c \\
b & a & a & c & a & c \\
c & a & a & c & a & c \\
d & d & d & e & d & e \\
e & d & d & e & d & e
\end{array} \\
& \leq=\{(a, a),(a, b),(a, c),(a, d),(a, e),(b, b),(b, c), \\
& (b, d),(b, e),(c, c),(c, e),(d, d),(d, e),(e, e)\} \text {. }
\end{aligned}
$$

The covering relation and the figure of $S$ are given by:

$$
<=\{(a, b),(a, c),(a, d),(a, e),(b, c),(b, d),(b, e),(c, e),(d, e)\}
$$

The right bases of $S$ are $\{e\}$ and $\{c\}$. The left bases of $S$ are $\{d\}$ and $\{e\}$. The two-sided bases of $S$ are $\{c\},\{d\}$ and $\{e\}$.

Lemma 4. Let $A$ be a subset of an ordered semigroup $(S, \cdot, \leq)$. Then $A$ is a right base of $S$ if and only if it satisfies the following conditions:

(i) for any $x$ in $S$ there exists $a$ in $A$ such that $L_{x} \preceq L_{a}$;

(ii) if $a, b \in A$ such that $a \neq b$, then neither $L_{a} \preceq L_{b}$ nor $L_{b} \preceq L_{a}$;

Proof. Assume that $A$ is a right base of $S$. Let $x \in S$. Since $S=(A \cup S A]$, so $x \in(A]$ or $x \in(S A]$. If $x \in(A]$, then $x \leq a$ for some $a$ in $A$; hence $L_{x} \preceq L_{a}$. If $x \in(S A]$, then $x \leq s a^{\prime}$ for some $s$ in $S$ and $a^{\prime}$ in $A$; hence $L_{x} \preceq L_{a^{\prime}}$. This proves that (i) holds. Let $a, b \in A$ such that $a \neq b$. Suppose $L_{a} \preceq L_{b}$. We set $B=A \backslash\{a\}$. Let $x \in S$. By (i), there exists $c$ in $A$ such that $L_{x} \preceq L_{c}$. If $c \neq a$, then $c \in B$; thus

$$
x \in L(x) \subseteq L(c) \subseteq(B \cup S B] .
$$

If $c=a$, then, by $b \in B$, we have 


$$
x \in L(x) \subseteq L(b) \subseteq(B \cup S B] .
$$

Thus $S=(B \cup S B]$. This is a contradiction. The case $L_{b} \preceq L_{a}$ is proved similarly. Thus (ii) holds true.

Conversely, assume that the conditions (i) and (ii) hold. By (i), $S=(A \cup$ $S A]$. Suppose that $S=(B \cup S B]$ for some a proper subset $B$ of $A$. Let $a \in A \backslash B$. If $a \leq b$ for some $b$ in $B$, then $L_{a} \preceq L_{a^{\prime}}$. This contradicts to (ii). Similarly, if $a \leq s b^{\prime}$ for some $s$ in $S$ and $b^{\prime}$ in $B$, then $L_{a} \preceq L_{b^{\prime}}$. This is a contradiction. Hence $A$ is a right base of $S$.

Theorem 5. Assume that an ordered semigroup $(S, \cdot, \leq)$ contains a left ideal. Then $L$ is a maximal left ideal of $S$ if and only if $S \backslash L$ is a maximal $\mathcal{L}$-class.

Proof. Assume that $L$ is a maximal left ideal of $S$. Let $x, y \in S \backslash L$. Since $L \subset L \cup L(x) \subseteq S$, we have $L \cup L(x)=S$, and so $y \in L(x)$. Similarly, $x \in L(y)$. Thus $L(x)=L(y)$. This proves that $S \backslash L$ is an $\mathcal{L}$-class. If $S \backslash L \prec L_{a}$ for some $a$ in $S$, then $S \backslash L \subseteq L(a) \subseteq L$. This is a contradiction. Therefore, $S \backslash L$ is a maximal $\mathcal{L}$-class.

Conversely, assume that $S \backslash L$ is a maximal $\mathcal{L}$-class such that $S \backslash L=L_{a}$ for some $a$ in $S$. If $x \in S L \backslash L$, then $x \in L_{a}$; hence $L(x)=L(a)$. By $x \in S L$, $x=s b$ for some $s$ in $S$ and $b$ in $L$. We have $L_{a} \prec L_{b}$. This is a contradiction. Hence $S L \subseteq L$. Let $y \in L$ and $c \in S$ be such that $c \leq y$. Suppose that $c \in L_{a}$. Then $c<y$, and so $L_{a} \prec L_{y}$. This is a contradiction. Thus $c \in L$. This shows that $L$ is a left ideal of $S$. Let $L^{\prime}$ be a left ideal of $S$ such that $L \subset L^{\prime} \subset S$. Then there is $z \in S \backslash L^{\prime}$. We have $L_{a}=L_{z}$. Similarly, there exists $w \in L^{\prime} \backslash L$ such that $L_{w}=L_{a}$. Then

$$
z \in L(z)=L(a)=L(w) \subseteq L^{\prime} .
$$

This is a contradiction. Therefore, $L$ is a maximal left ideal of $S$.

Corollary 6. If an ordered semigroup $(S, \cdot, \leq)$ contains a right base, then $S$ contains a maximal left ideal.

Proof. This is a consequence of Lemma 4 and Theorem 5.

Definition 7. A proper left ideal $L$ of an ordered $\operatorname{semigroup~}(S, \cdot, \leq)$ is called a covered left ideal if $L \subseteq(S(S \backslash L)]$.

Example 8. ([8]) Let $(S, \cdot, \leq)$ be an ordered semigroup such that $S=$ $\{a, b, c, d, e\}$ and 


$$
\begin{aligned}
& \begin{array}{c|ccccc}
\cdot & a & b & c & d & e \\
\hline a & a & b & a & a & a \\
b & a & b & a & a & a \\
c & a & b & a & a & a \\
d & a & b & a & a & a \\
e & a & b & a & a & e
\end{array} \\
& \leq=\{(a, a),(a, b),(a, e),(b, b),(c, b),(c, c),(c, e),(d, d),(d, b),(d, e),(e, e)\} .
\end{aligned}
$$

The covering relation and the figure of $S$ are given by:

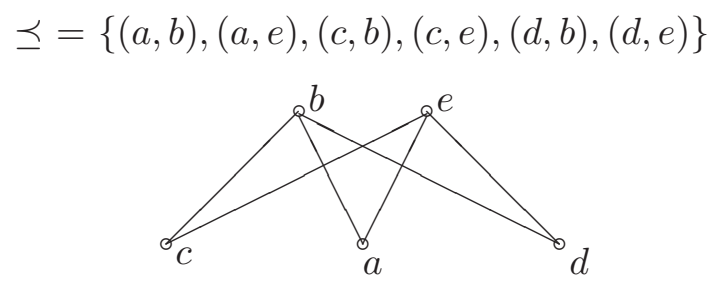

The left ideals of $S$ are $\{a\},\{a, c\},\{a, d\},\{a, c, d\},\{a, b, c, d\},\{a, c, d, e\}$ and $S$. The covered left ideals of $S$ are $\{a\},\{a, c\},\{a, d\},\{a, c, d\}$.

Example 9. ([5]) Let $(S, \cdot, \leq)$ be an ordered semigroup such that $S=\{a, b, c, d, f\}$ and

$$
\begin{aligned}
& \begin{array}{c|lllll}
\cdot & a & b & c & d & e \\
\hline a & b & d & a & b & e \\
b & d & b & b & d & e \\
c & d & b & c & d & e \\
d & b & d & d & b & e \\
e & e & e & e & e & e
\end{array} \\
& \leq=\{(a, a),(b, b),(b, c),(b, e),(c, c),(d, a),(d, d),(d, e),(e, e)\} .
\end{aligned}
$$

The covering relation and the figure of $S$ are given by:

$$
\preceq=\{(b, c),(b, e),(d, a),(d, e)\}
$$

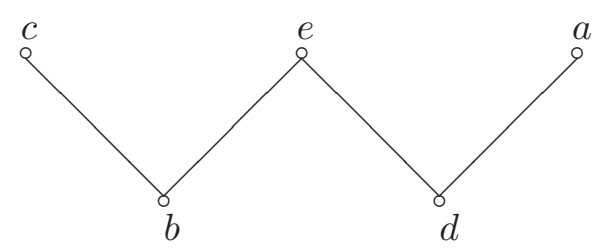


The left ideals of $S$ are $\{b, d, e\},\{a, b, d, e\}$ and $S$. The covered left ideal of $S$ is $\{b, d, e\}$.

Corollary 10. Let $(S, \cdot, \leq)$ be an ordered semigroup containing a right base. Then the following statements hold.

(1) $S$ contains maximal left ideals.

(2) Every maximal left ideal $L_{i}$ of $S$ is $L_{i}=S \backslash L_{a_{\mathrm{i}}}$ for some $a_{i}$ in $S$.

Proof. It is a consequence of Theorem 4 and 5 .

Theorem 11. Let $(S, \cdot, \leq)$ be an ordered semigroup containing maximal left ideals. If the intersection of all maximal left ideals of $S$ is empty or a covered left ideal, then $S$ contains a right base.

Proof. Let $\left\{L_{i} \mid i \in I\right\}$ be the set of all maximal left ideals of $S$. By Theorem 5 , for each $i \in I, S \backslash L_{i}$ is a maximal $\mathcal{L}$-class. Setting

$$
S \backslash L_{i}:=L_{a_{\mathrm{i}}} \text { for each } i \text { in } I
$$

then

$$
L:=\bigcap_{i \in I} L_{i}=\bigcap_{i \in I}\left(S \backslash L_{a_{\mathrm{i}}}\right)=S \backslash \bigcup_{i \in I} L_{a_{\mathrm{i}}} .
$$

Let $A$ denote the set of all elements $a_{i}$. We assert that $A$ is a right base of $S$, and hence the theorem is proved. We consider two cases:

Case 1: $L=\emptyset$. Then $S=\bigcup_{i \in I} L_{a_{\mathrm{i}}}$. If $x \in S$, then $x \in L_{a_{\mathrm{i}}}$ for some $i$ in $I$, and so $L(x)=L\left(a_{i}\right)$. Thus $L_{x} \preceq L_{a_{\mathrm{i}}}$. Since $L_{a_{\mathrm{i}}}$ is a maximal $\mathcal{L}$-class for all $i$ in $I$, it follows that, for any different $i, j$ in $I$, neither $L_{a_{\mathrm{i}}} \preceq L_{a_{\mathrm{j}}}$ nor $L_{a_{\mathrm{j}}} \preceq L_{a_{\mathrm{i}}}$. By Lemma $4, A$ is a right base of $S$.

Case 2: $L$ is a covered left ideal of $S$. That is, $L \subseteq(S(S \backslash L)]$. If $x \in S \backslash L$, then $x \in \bigcup_{i \in I} L_{a_{\mathrm{i}}}$, and so $x \in L_{a_{\mathrm{i}_{0}}}$ for some $i_{0}$ in $I$. Since

$$
L(x)=L\left(a_{i_{0}}\right) \subseteq(A \cup S A],
$$

we have $x \in(A \cup S A]$. This proves that

$$
S \backslash L \subseteq(A \cup S A] .
$$

By

$$
L \subseteq(S(S \backslash L)] \subseteq(S(A \cup S A)] \subseteq(S A \cup S S A] \subseteq(A \cup S A]
$$

it follows that 


$$
S=L \cup(S \backslash L) \subseteq(A \cup S A] .
$$

This implies that if $x \in S$ then there exists $a_{i} \in A$ such that $L_{x} \preceq L_{a_{\mathrm{i}}}$. It follows by Lemma 4 that $A$ is a right base of $S$.

It is not true in general that an ordered semigroup contains one-sided bases implies the semigroup contains two-sided bases. This was shown by Example 2 in [2]. However, it is easy to see that this statement holds true for any finite ordered semigroups.

Theorem 12. If an ordered semigroup $(S, \cdot, \leq)$ contains a left or a right base which is finite, then $S$ contains a two-sided base.

Corollary 13. Every finite ordered semigroup contains both one-sided bases and two-sided bases.

\section{References}

[1] G. Birkhoff, Lattice theory, 25, Rhode Island, American Mathematical Society Colloquium Publications, Am. Math. Soc., Providence, 1984.

[2] I. Fabrici, On bases and maximal ideals in semigroups, Mathematica Slovaca, 31 (1981), 115-120.

[3] N. Kehayopulu, On weakly prime ideals of ordered semigroups, Math. Japonica, 35(6) (1990), 1051-1056.

[4] N. Kehayopulu, On completely regular ordered semigroups, Scinetiae Mathematicae, 1(1) (1998), 27-32.

[5] N. Kehayopulu, M. Tsingelis, A note on ordered groupoids-semigroups, Scientiae Mathematicae, 3 (2000), 251-255.

[6] N. Kehayopulu, M. Tsingelis, On left regular ordered semigroups, Southeast Asian Bulletin of Mathematics, 25 (2002), 609-615.

[7] N. Kehayopulu, M. Tsingelis, On subdirectly irreducible ordered semigroups, Semigroup Forum, 50 (1995), 161-177.

[8] N. Kehayopulu, M. Tsingelis, On weakly commutative ordered semigroups, Semigroup Forum, 56 (1998), 32-36.

[9] T. Tamura, One sided-bases and translations of a semigroup, Math. Japan, 3 (1955), 137-141. 